\title{
Angeborene Lungenerkrankungen - welche Diagnostik ist wann sinnvoll?
}

Fehlbildungen der Lungen und des Tracheobronchialsystems sind bei entsprechend wahrgenommenen Vorsorgeuntersuchungen in der Schwangerschaft oft pränatal bekannt. Sonografisch sind bei ausreichender Expertise die meisten Erkrankungen sicher diagnostizierbar. Mit der fetalen MRT steht der Kinderradiologie eine weiterführende Bildgebung zur Verfügung, die zunehmend angewandt wird - bei Diskrepanz der Ultraschallbefunde und zur Verifizierung bzw. Prognoseabschätzung (Lungenreife, Thorax-Konfiguration, assoziierte Fehlbildungen, Fruchtwassermenge) ist sie indiziert. Postnatal ist die Röntgenübersichtsaufnahme in
1 Ebene Methode der ersten Wahl, ergänzt um die Sonografie zur Beurteilung pleuraler und Thoraxwand-naher Lungenveränderung bzw. Abklärung radiografisch auffälliger Befunde. Die Indikation zur CT oder MRT ist individuell nach interdisziplinärer Diskussion zu stellen - die Durchführung ist abhängig von den lokalen Gege-
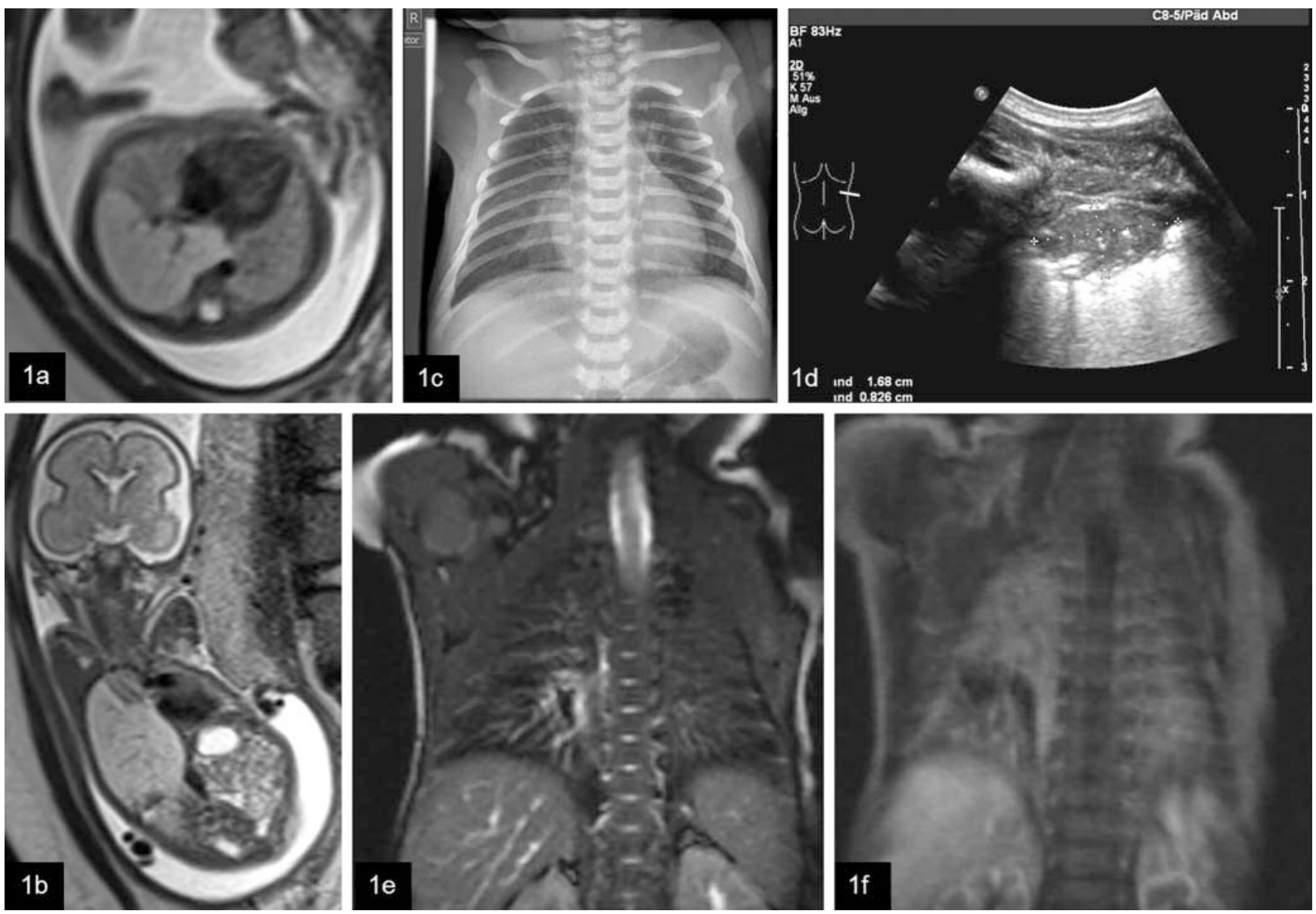

- Abb. 1 CPAM Typ III nach Stocker. a Fetale MRT axial; b fetale MRT koronar; c postnatales Röntgen; d Sonografie; e MRT T2w koronar; f MRT T1w koronar post KM. 

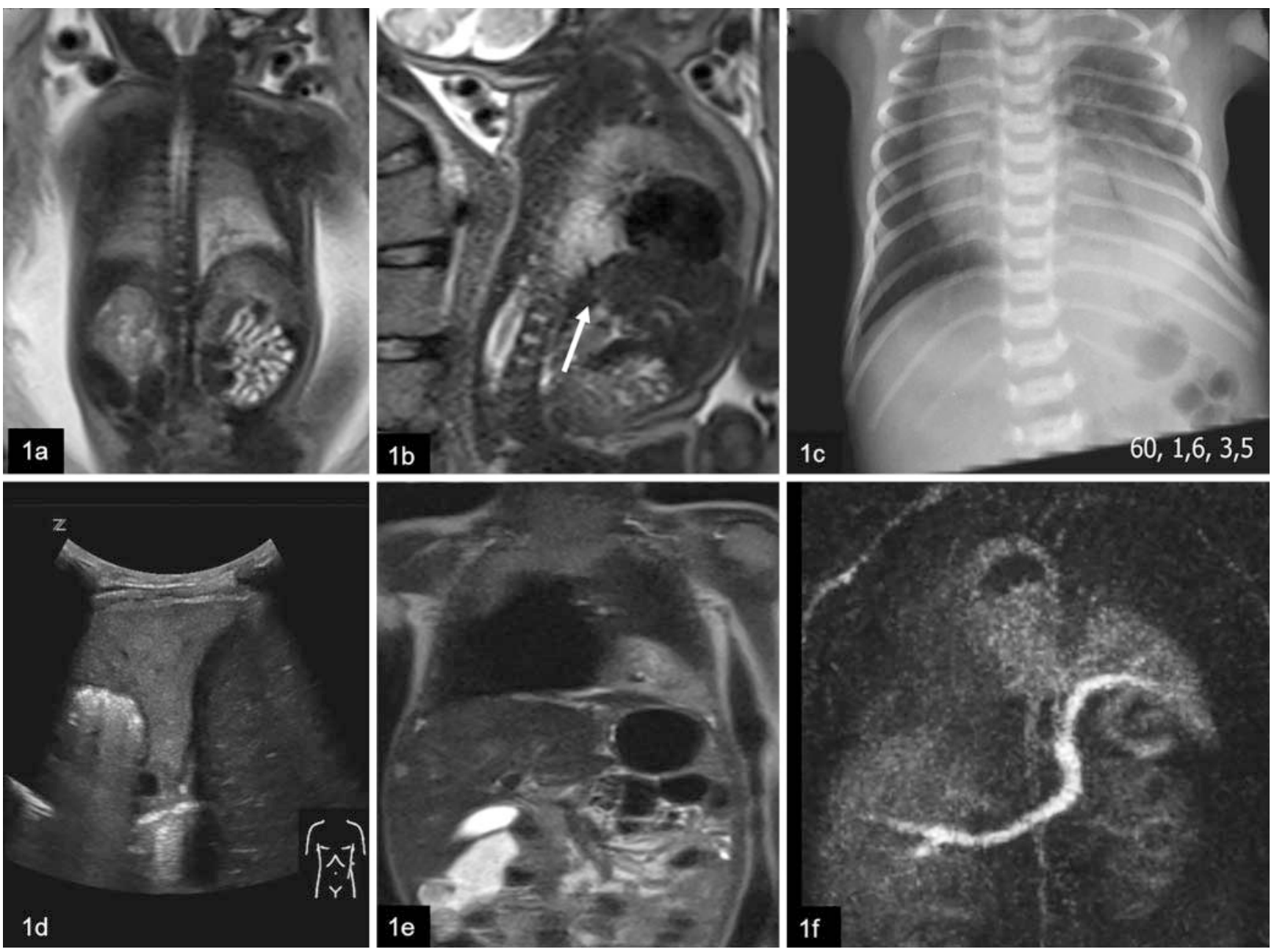

- Abb. 2 Lungensequester. a Fetale MRT koronar; b fetale MRT sagittal (Pfeil markiert Gefäß aus Aorta abdominalis); c postnatales Röntgen; d Sonografie echoreicher Sequester; e MRT T2w koronar, Sequester hyperintens; f MRT T1w koronar, venöse Drainage in die Vena portae.

benheiten; je später, umso reifer ist der Säugling, umso eher kann Kontrastmittel appliziert werden. Mitunter sind günstige Untersuchungsbedingungen wie in einem MR-Inkubator dann aber nicht mehr gegeben, alternativ können die Feed-andWrap-Technik oder spezielle Wickel- und Vakuumtechniken verwendet werden, um den Kindern eine Narkose zu ersparen.

Lungendysmorphie, Lungenagenesie und Lungenaplasie sind Hemmungsfehlbildungen, die bereits pränatal diagnostiziert werden. Bei einseitiger Lungenagenesie ist postnatal im Röntgen-Thorax die betroffene Thorax-Hälfte komplett verschattet, die Rippen stehen eng und die gesunde Seite ist entsprechend kompensatorisch überbläht und vermehrt transparent. CT und Bronchoskopie bestätigen die Befunde.
Lungenhypoplasie wird definiert als Zustand, bei dem <60\% des erwarteten Lungenvolumens bestehen. Beobachtet wird sie bei An-/Oligohydramnion (häufig renal bedingt), Hydrothorax sowie bei vorzeitigem Blasensprung. Postnatal besteht typisch ein schweres Atemnotsyndrom, der Röntgen-Thorax zeigt eine Glockenform, kleine Lungen, hochstehendes Zwerchfell.

Congenital pulmonary airway malformation (CPAM) - früher als Congential cystic adenomatoid malformation (CCAM) bekannt - ist die häufigste angeborene Lungenfehlbildung ( $\mathbf{A}$ bb. 1). Sie kann meist bereits pränatal klassifiziert werden; es können 5 Subtypen unterschieden werden. Nach Stocker werden bildmorphologisch 3 Typen eingeteilt (Typ $1-65 \%-2-10 \mathrm{~cm}$ große Zysten; Typ 2 -10-40\% -0,5-2 cm große Zysten; Typ $3-<0,5 \mathrm{~cm}$ Zysten, eher solid- schwammartiger Aspekt in der Bildgebung). Zielführend ist die T2-wichtende fetale MRT mit langer Echozeit (> $100 \mathrm{~ms}$ ). Prognostisch ungünstig sind raumfordernd große Läsionen, Mediastinalshift, Polyhydramnion, Hydrops fetalis. Eine spontane Rückbildung in der Schwangerschaft ist möglich (nach 25. SSW). Postnatal ist auch bei asymptomatischem Kind der Röntgen-Thorax indiziert (primär weichteildicht, später Aufhellung zystischer Anteile); CT-Untersuchung nach (möglichst) > 6 Wochen mit Kontrastmittelgabe zur Abschätzung der Ausdehnung der Veränderungen, MRT bei entsprechender Technik möglich; zwar sind die postnatal zystisch-luftgefüllten Veränderungen nicht so gut klassifizierbar, die Abgrenzung zum Sequester ist in der MRT sicherer. Operative Entfernung (maligne Entartung beschrieben) bzw. die Frequenz von Kontrolluntersuchungen (Röntgen/MRT) werden diskutiert. 


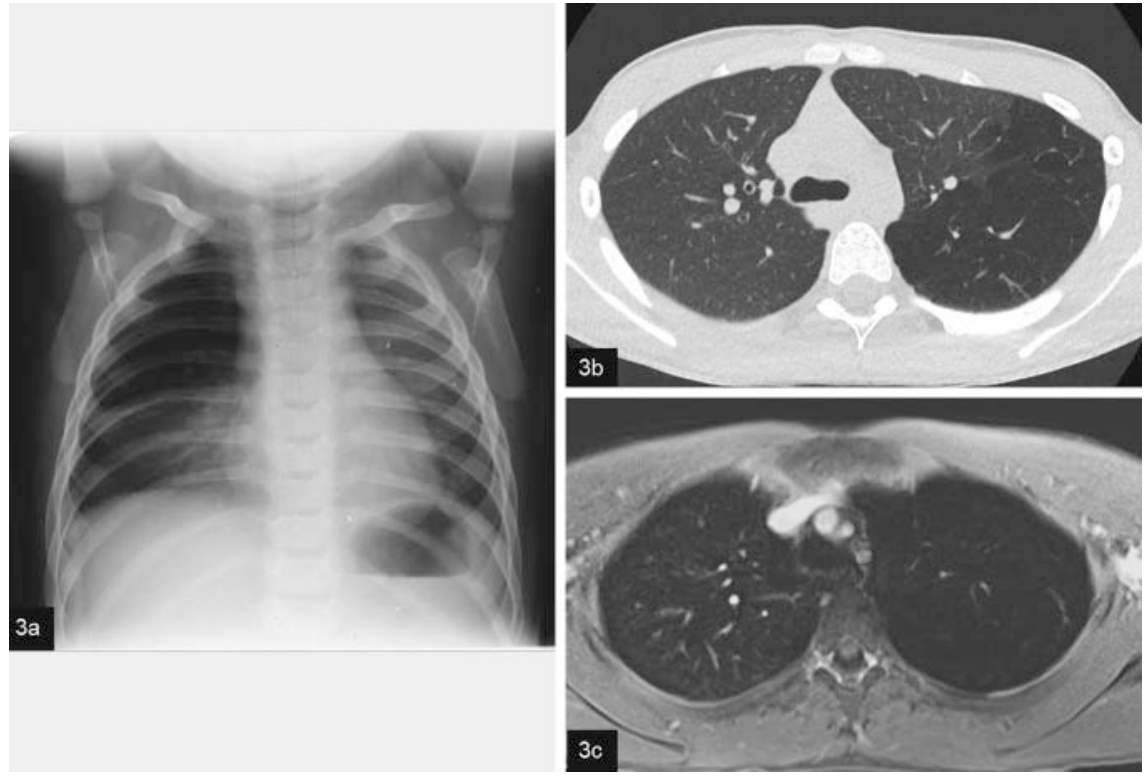

- Abb. 3 Kongenitales lobäres Emphysem. a Röntgenbild mit vermehrter Transparenz rechts im Oberlappenbereich; $\mathbf{b}$ CT im Lungenfenster mit vermehrter Transparenz bei rarefizierter Lungengefäßzeichnung links im Unterlappenbereich; c MRT T1w nach Kontrastmittelapplikation mit verminderter Lungengefäßzeichnung links im Oberfeld bei Emphysem.

Bronchopulmonale Sequester sind per definitionem funktionslose Lungenteile und die zweithäufigsten pränatal diagnostizierten Lungenfehlbildungen ( $\triangleright$ Abb. 2). Intralobäre Sequester werden aus der Aorta abdominalis versorgt und drainieren in Pulmonalvenen, extralobäre in die Systemvenen (VCI/V. azygos, V. portae). Sonografisch imponiert der Sequester echoreicher, in der fetalen MRT oft signalärmer in T2w und hyperintens in T1w im Vergleich zum Lungenparenchym. Diagnostisch ist in der T2w das signalfreie zuführende Gefäß aus der Aorta thoracalis/abdominalis (Schichtdicke $\leq 4 \mathrm{~mm}$ ). Postnatal sind der Röntgen-Thorax (Verschattung) und die B-Mode- und Farbdoppler-Sonografie indiziert; zu differenzieren sind infradiaphragmale Sequester von Nebennierenläsionen. Bei unklaren Befunden kann die MRT (ggf. mit Kontrastmittelgabe ab der 6. Lebenswoche) der CT vorgezogen werden.
Bronchusatresie ist im fetalen MRT durch ein stark T2w-hyperintenses Signal des nachgeschalteten Lungenabschnitts bei erhaltener Gefäßarchitektur gekennzeichnet und schwer abzugrenzen von der CPAM Typ 3.

Kongenitales lobäres Emphysem ist durch Überblähung eines oder mehrerer Lungenlappen definiert ( $\triangleright$ Abb. 3). Pränatal ist das betroffene Parenchym T2whyperintens (Lungenzysten sind stark hyperintens). Polyhydramnion, Hydrops fetalis und Lungenhypoplasie sind möglich und bedeuten eine schlechtere Prognose. Unmittelbar postnatal kann der Befund meist nicht erfasst werden. „Später“ besteht eine Überblähung mit rarefizierter Gefäßzeichnung, möglicherweise mit Verdrängung der Nachbarschaft (Zwerchfell, Lappenspalt, Mediastinum, Herz) und evtl. Kompression angrenzender Lungenabschnitte (Dys-/Atelektase). Die CT verifi- ziert den Befund und ist präoperativ indiziert; prinzipiell ist Kontrastmittel nicht erforderlich.

Kongenitale Lymphangiektasie ist sehr selten, tritt sporadisch auf und ist durch subpleurale, interlobäre, perivaskuläre und peribronchiale Lymphgefäßerweiterung gekennzeichnet. Ein Chylothorax (Sonografie) kann zur Verdachtsdiagnose führen. Die erweiterten Lymphgefäße entgehen postnatal meist dem Nachweis im Röntgen; die CT kann Milchglastrübung, verdickte Septen und bronchovaskuläre Bündel im Lungenfenster zeigen.

\section{Interessenkonflikt}

Die Autoren geben an, dass kein Interessenkonflikt besteht.

Autorinnen/Autoren

Hans-Joachim Mentzel

Universitätsklinikum Jena, Sektion Pädiatrische Radiologie, Institut für Diagnostische und Interventionelle Radiologie

\section{Korrespondenzadresse}

Univ.-Prof. Dr. med. Hans-Joachim Mentzel Universitätsklinikum Jena Sektion Pädiatrische Radiologie Institut für Diagnostische und Interventionelle Radiologie Am Klinikum 1

07747 Jena

Hans-joachim.mentzel@med.uni-jena.de

\section{Bibliografie}

DOI https://doi.org/10.1055/a-0943-1293 Online-Publikation: 2019

Fortschr Röntgenstr 2019; 191: S119-S121

(c) Georg Thieme Verlag KG, Stuttgart · New York ISSN 1433-5972 